\title{
Utillità della biopsia epatica mella valutazione d'idoneità al trapianto di rene del paziente con infezione da virus C
}

\author{
N. Bossini ${ }^{1}$, S. Sandrini ${ }^{1}$, L. Bercich ${ }^{2}$, G. Setti ${ }^{1}$, S. Turina ${ }^{1}$, F. Londrino ${ }^{1}$, G. Cancarini ${ }^{1}$ \\ ${ }^{1}$ Divisione e Cattedra di Nefrologia \\ ${ }^{2}$ Servizio di Anatomia Patologica I degli Spedali Civili e Università di Brescia, Brescia
}

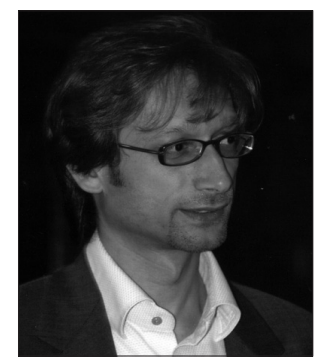

N. Bossini
I infezione da HCV rappresenta ancor oggi un problema di grande rilevanza clinica ed epidemiologica per i pazienti con insufficienza renaalta prevalenza e delle sue potenzial implicazioni nel post-trapianto renale Per quanto l'incidenza di sieropositività anti-HCV nella popolazione di uremici dei Centri Dialisi in Italia si sia progressivamente ridotta nell'ultimo decennio, ancor oggi la prevalenza si attesta mediamente intorno al 15$20 \%$ e l'infezione da virus C rimane la principale causa di malattia epatica cronica e la quarta causa di morte tra $\mathrm{i}$ riceventi di trapianto renale (1).

Il rischio di una più rapida evoluzione dell'epatite $\mathrm{HCV}$ correlata conseguente all'immunosoppressione farmacologica è ancora controverso, tuttavia, la progressione dell'epatopatia sembra dipendere dalla severità delle lesioni istologiche presenti al momento del trapianto (2). Per questo motivo, da alcuni anni, è stato proposto di inserire

lo studio morfologico del danno epatico tra i parametri da utilizzare per la valutazione del paziente $\mathrm{HCV}$ positivo canditato al trapianto di rene (3). Sebbene questa strategia abbia trovato largo consenso da parte di molti autori, tuttora non esistono dati che abbiano documentato la sua effettiva utilità in questo specifico ambito.

Nel nostro Centro, da circa 10 anni, viene consigliata l'esecuzione di una biopsia epatica a pazienti HCV positivi viremici, allo scopo di associare alla valutazione clinica anche quella istologica.

Obiettivo di questo studio è stabilire se l'esecuzione di una biopsia epatica pre-trapianto permetta una selezione più accurata del paziente $\mathrm{HCV}$ positivo rispetto ai parametri clinici e di laboratorio comunemente utilizzati.

\section{PAZIENTI E METODI}

Criteri di selezione della popolazione in studio. Sono stati esaminati retrospettivamente tutti i pazienti HCV-ab positivi in terapia dialitica, valutati per inserimento in lista di trapianto di rene presso il Centro Trapianti della Divisione di Nefrologia degli Spedali
Civili di Brescia, dal $1^{\circ}$ gennaio 1990 al 31 luglio 2005. La coinfezione con virus B e l'assenza di dati clinici e bioumorali certi, nell'anno precedente alla valutazione, sono stati considerati criteri di esclusione.

Dati anagrafici, anamnestici, clinici e virologici. Al momento della valutazione, per ogni paziente sono state registrate le seguenti informazioni: sesso, età, eziologia della malattia renale, durata e tipo di dialisi, pregresse emotrasfusioni, precedenti trapianti, tossicodipendenza, stima della durata dell'infezione da HCV (desunta dalla data della prima esposizione a un fattore di rischio), presenza di segni clinici di cirrosi e/o scompenso epatico (quadro ecografico di cirrosi, ipertensione portale, ascite, varici esofagee, encefalopatia), dosaggio qualitativo o quantitativo di HCV-RNA e tipizzazione genomica del virus $\mathrm{C}$, dosaggio delle transaminasi (misurate almeno due volte nel corso dell'anno precedente alla valutazione di idoneità al trapianto), albuminemia e tempo di protrombina (PT). È stato considerato patologico qualsiasi valore di transaminasi al di sopra del range di normalità; il riscontro di ipertransaminasemia, anche se lieve e transitorio, defi- 


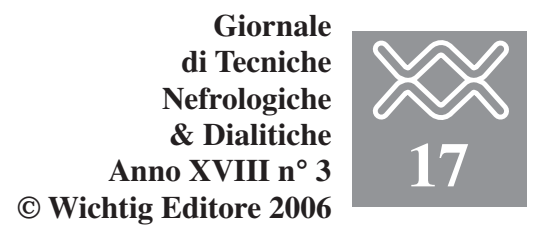

niva la presenza di epatocitolisi.

Biopsia epatica. L'indicazione alla biopsia veniva posta in seguito al riscontro di HCV-RNA positività, indipendentemente dalla presenza o meno di epatocitolisi. Ogni biopsia epatica veniva eseguita sotto guida ecografica con ago $21 \mathrm{G}$, previo consenso del paziente, dopo aver verificato la normalità dei parametri coagulativi standard (PT, PTT, Fibrinogeno), della conta piastrinica e del tempo di sanguinamento (tempo di Ivy).

Il danno istologico era classificato utilizzando uno score descrittivo (Tab. I). Analisi statistica. Dopo una prima valutazione statistica descrittiva delle variabili considerate, sono stati utilizzati i seguenti test:

$\mathrm{t}$ di Student per dati appaiati e non appaiati Test 2 .

L'archiviazione dei dati è stata seguita con il programma "Excel" ${ }^{\circledR}$ di Microsoft; l'analisi statistica con i programmi statistici di Excel ${ }^{\circledR}$. È stata accettata come significatività una $\mathrm{p}<0.05$.

\section{RISUTATI}

Caratteristiche della popolazione studiata

Su 1399 pazienti valutati per idoneità al trapianto di rene dal gennaio 1990 al luglio 2005, 194 sono risultati HCV-Ab positivi (13.8\%). Dopo l'esclusione di 16 pazienti che erano coinfettati da virus dell'epatite B e di 22 pazienti di cui non si erano acquisi- ti dati certi sulla viremia e/o sull'andamento delle transaminasi nel corso dell'anno precedente alla valutazione, la popolazione inclusa nello studio è risultata costituita da 156 pazienti $\mathrm{HCV}-\mathrm{Ab}$ positivi, con età media di $43 \pm 11$ anni, 93 (60\%) maschi e 63 (40\%) femmine.

La popolazione HCV-Ab positiva, rispetto ai pazienti $\mathrm{HCV}-\mathrm{Ab}$ negativi, era caratterizzata da un'età inferiore, da una durata di dialisi maggiore, da una maggior frequenza di trasfusioni e di pregressi trapianti. La condizione di $\mathrm{HCV}-\mathrm{Ab}$ positività era più frequente nella popolazione in dialisi extracorporea rispetto a quella in dialisi peritoneale (Tab. II).

Tra i 156 pazienti HCV-Ab positivi segni clinici e/o strumentali di cirrosi e/o insufficienza epatica sono stati rilevati in un solo caso $(0.6 \%)$. In $40 / 156(25.6 \%)$ pazienti era presente un incremento persistente o transitorio delle transaminasi. La ricerca dell'HCV-RNA è stata effettuata in $131 / 156(84 \%)$ pazienti ed è risultata positiva in $102(78 \%)$. Il genotipo è stato studiato in 63/102 (62\%) pazienti. I genotipi più frequenti sono risultati l'1b (49.2\%) ed il 2a/2c (36.5\%). Non sono state riscontrate correlazioni significative tra genotipo ed epatocitolisi (Tab. III).

\section{Biopsia epatica}

Sono stati sottoposti a biopsia epatica 63 pazienti: $26(41 \%)$ di questi presentavano sia viremia che citolisi, 37
$(59 \%)$ solo viremia. L'intervallo di tempo intercorrente tra l'inizio della dialisi (considerato come inizio dell'infezione da virus C) e la data di esecuzione della biopsia epatica, era mediamente di $68 \pm 64$ mesi. Solo una biopsia ecoguidata sulle 63 effettuate $(1.6 \%)$ è stata complicata da un emoperitoneo. Tale complicanza si è verificata in un paziente in dialisi peritoneale e ha avuto scarsa rilevanza clinica, condizionando solamente un tempo di osservazione post-bioptico di 24 ore. L'esito dell'esame istologico è riassunto nella Figura 1. Il quadro più frequentemente osservato è stato l'epatite cronica attiva (ECA) minima/lieve $(62 \%)$. Solo in due pazienti $(3 \%)$ è stata riscontrata un'ECA moderata e in nessun caso quadri di ECA severa e cirrosi. In tutte le biopsie il grado di fibrosi era lieve o assente. Epatocarcinoma nell'ambito di un'ECA lieve è stato riscontrato in 1 caso.

Confronto tra presenza o meno di epatocitolisi e quadro istologico

Pazienti senza citolisi. Sono 37 pazienti tutti viremici. L'esame istologico in questo gruppo di pazienti (Tab. IV) ha mostrato quadri di normalità e di epatite aspecifica o comunque non aggressiva in 15 casi $(40.5 \%)$, ed ECA minima/lieve in $22(59.5 . \%)$ tra cui un caso con associato epatocarcinoma per il quale era stato posto un sospetto ecografico. Non sono stati riscontrati casi di ECA moderata o severa né ad evoluzione cirrogena (Tab. IV).

\section{TABELLA I - DIAGNOSI DESCRITTIVA UTILIZZATA PER CLASSIFICARE I RISULTATI DELLA BIOPSIA EPATICA}

Lesioni epatiche aspecifiche (LEA) Epatite cronica persistente (ECP) Epatite cronica aggressiva minima (ECA minima)

Epatite cronica aggressiva moderata (ECA moderata)

Epatite cronica aggressiva severa (ECA severa)

Fibrosi lieve

Fibrosi severa

Cirrosi

Siderosi
Epatite senza piecemeal necrosis e necrosi lobulare minima Epatite senza piecemeal necrosis

Epatite con piecemeal necrosis focale in alcuni spazi portali

Epatite con piecemeal necrosis continua coinvolgente meno del $50 \%$ del perimetro degli spazi portali e/o setti

Epatite con piecemeal necrosis continua coinvolgente più del $50 \%$ del perimetro degli spazi portali e/o setti

Fibrosi degli spazi portali

Fibrosi a ponte porto-portale e/o porto-centrale

Epatite con presenza di setti e atteggiamento nodulare focale e/o incompleto del parenchima presenza di granuli intraepatocitari e/o macrofagici 
TABELLA II - CARATTERISTICHE DELLA POPOLAZIONE HCV ${ }^{+}$STUDIATA; CONFRONTO CON LA POPOLAZIONE HCV

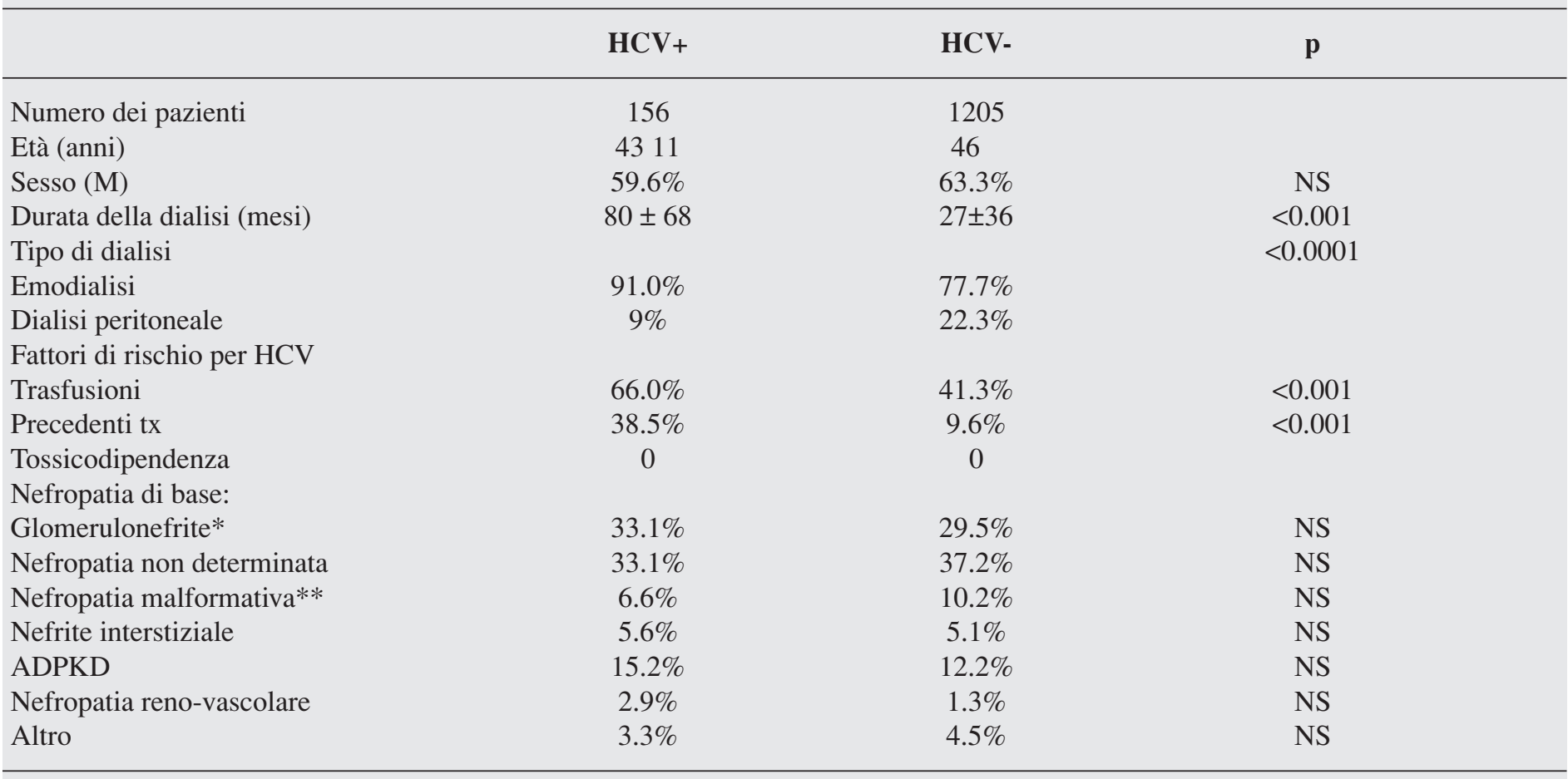

*Glomerulonefriti sia primitive che secondarie diagnosticate istologicamente

**Compreso reflusso $\mathrm{v}-\mathrm{u}$

TABELLA III - DISTRIBUZIONE DEI GENOTIPI NELLE POPOLAZIONI DI PAZIENTI RISPETTIVAMENTE CON CITOLISI E SENZA CITOLISI

\begin{tabular}{lccc}
\hline Genotipo & Paz con citolisi (19) & Paz senza citolisi (44) & p \\
\hline 1 & $57.9 \%$ & $59.1 \%$ & NS \\
$2 \mathrm{a} / 2 \mathrm{c}$ & $36.8 \%$ & $36.4 \%$ & NS \\
altri & $5.3 \%$ & $4.5 \%$ & NS \\
\hline
\end{tabular}

\section{Pazienti con citolisi}

Si tratta di 26 pazienti con riscontro persistente o occasionale di ipertransaminasemia. I quadri istologici riscontrati in questi pazienti sono risultati molto variabili (Tab. IV). Diciannove su 26 pazienti $(73.1 \%)$ presentavano un quadro istologico di ECA, ma nella maggior parte dei casi l'attività necroinfiammatoria era minima/lieve e solo in 2 casi $(7.7 \%)$ risultava moderata. Anche in questo gruppo di pazienti non sono stati osservati quadri
TABELLA IV - ALTERAZIONI ISTOPATOLOGICHE IN 63 PAZIENTI

\begin{tabular}{lcc}
\hline & No citolisi (37) & Citolisi (26) \\
\hline Normalità & $3(8.1 \%)$ & 0 \\
Epatosidereosi & 0 & $2(7.7 \%)$ \\
Epatite aspecifica reattiva & $9(24.3 \%)$ & $2(7.7 \%)$ \\
Epatite cronica persistente & $3(8.1 \%)$ & $3(11.5 \%)$ \\
Epatite cronica attiva lieve & $22 *(59.5 \%)$ & $17(65.4 \%)$ \\
Epatite cronica attiva moderata & 0 & $2(7.7 \%)$ \\
Epatite cronica attiva severa & 0 & 0 \\
\hline
\end{tabular}

* Un paziente con $\mathrm{HCC}$ 
di ECA severa o a evoluzione cirrogena. La distribuzione dei vari gradi di epatopatia non differisce in modo statisticamente significativo tra i pazienti con e senza citolisi.

Confronto tra pazienti con ECA e quelli senza ECA

I pazienti con diagnosi istologica di epatite cronica aggressiva, con qualsiasi grado di attività, sono stati confrontati con i pazienti con quadro istologico aspecifico o di normalità. Da tale confronto non è emerso alcun tipo di correlazione né di tipo clinicoanamnestico né di tipo virologico (Tab. V).

Idoneità al trapianto di rene dopo biopsia epatica

Su 63 pazienti studiati con biopsia epatica, solamente $2(3 \%)$ presentavano un quadro di epatite cronica attiva di gravità sufficiente a porre controindicazione al trapianto di rene. Si trattava dei 2 pazienti con ECA moderata. Entrambi presentavano una storia di epatocitolisi. In entrambi è stata posta indicazione a trattamento antivirale. In nessun paziente senza citolisi epatica la biopsia rivelava forme di epatite di grado ed evoluzione tali da controindicare il trapianto.

\section{DISCUSSIONE}

I dati di prevalenza dell'infezione da virus $\mathrm{C}$ nella popolazione da noi studiata sono sovrapponibili a quanto già descritto nella maggior parte dei lavori pubblicati (4). Allo stesso modo, anche nella nostra popolazione si confermano le note correlazioni tra epatite $\mathrm{C}$ e fattori di rischio quali, la dialisi extracorporea, le emotrasfusioni e i precedenti trapianti.

Il rischio teorico di una più rapida progressione dell'epatite $\mathrm{C}$ indotta dall'immunosoppressione, la sostanziale impossibilità a utilizzare l'interferone dopo trapianto per l'elevato rischio di rigetto acuto (5), la correlazione tra severità delle lesioni istologiche pre-trapianto e l'evolutività dell'epatopatia (2) e l'assenza di una

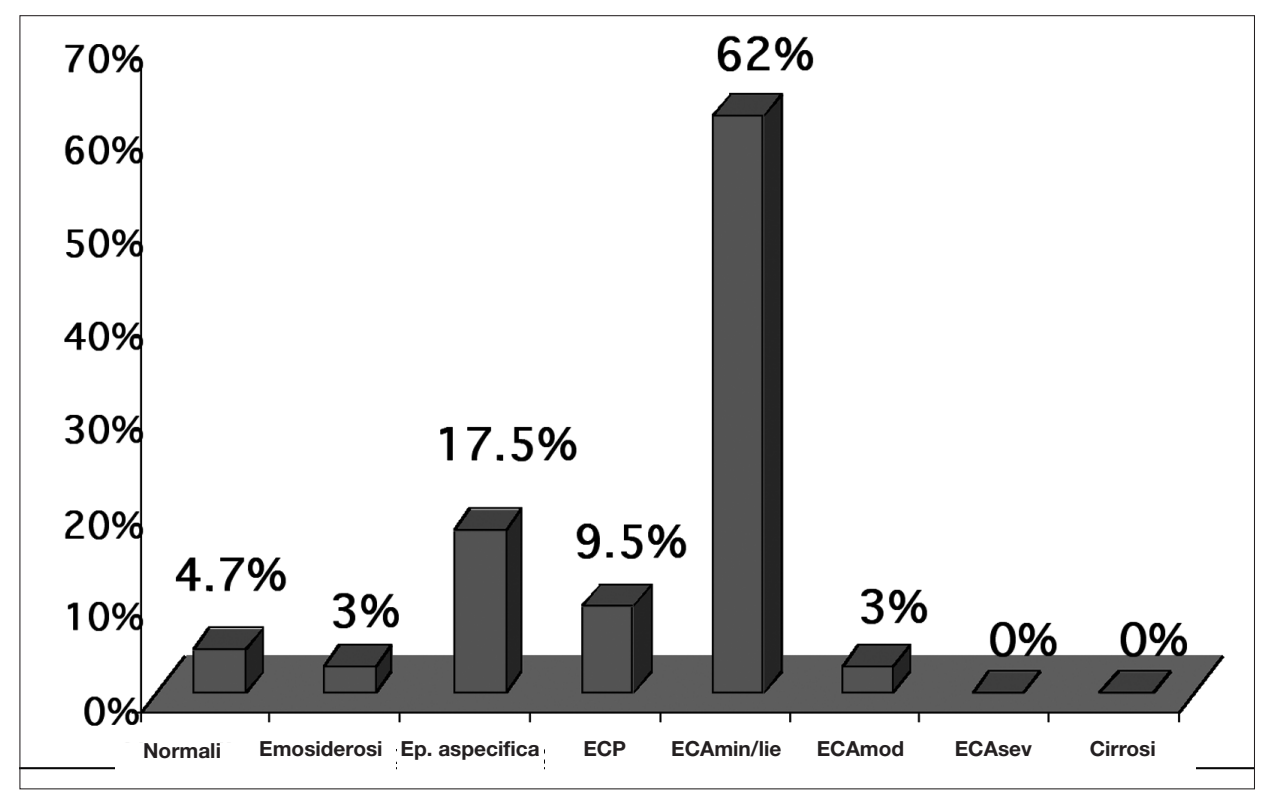

Fig. 1 - Prevalenza delle lesioni istologiche in tutte le biopsie eseguite. ECP: epatite cronica persistente; ECAmin/lie: epatite cronica aggressiva minima/lieve; ECAmod: epatite cronica aggressiva moderata; ECAsev: epatite cronica aggressiva severa.

\section{TABELLA V - CONFRONTO TRA PAZIENTI CON ECA E QUELLI CON LESIO- NI EPATICHE ASPECIFICHE, EMOSIDEROSI O QUADRO DI NORMALITÀ}

\begin{tabular}{lccc}
\hline & $\begin{array}{r}\text { Pazienti } \\
\text { con ECA }\end{array}$ & $\begin{array}{c}\text { Pazienti } \\
\text { senza ECA }\end{array}$ & p \\
\hline $\mathrm{N}^{\circ}$ pazienti & 41 & 22 & \\
Età media (anni) & $44 \pm 10$ & $44 \pm 13.6$ & $\mathrm{NS}$ \\
Durata dialisi (mesi) & $50 \pm 44$ & $71 \pm 66$ & $\mathrm{NS}$ \\
Citolisi $\mathrm{N}^{\circ} \%$ ) & $46.4 \%$ & $31.8 \%$ & $\mathrm{NS}$ \\
Tipo di dialisi (\%HD) & $90 \%$ & $100 \%$ & $\mathrm{NS}$ \\
Genotipi: & $10 \%$ & 0 & \\
$\quad$ 1a & $53 \%$ & $50 \%$ & $\mathrm{NS}$ \\
1b & $30 \%$ & $50 \%$ & $\mathrm{NS}$ \\
2a/2c & $6.6 \%$ & 0 & \\
altri & & & \\
\hline
\end{tabular}

precisa correlazione tra dati biochimici e quadri istologici (6), hanno portato a suggerire la biopsia epatica come strumento utile per una corretta valutazione di idoneità al trapianto renale del paziente uremico con infezione da virus C. Tuttavia, non sono ancora disponibili dati definitivi che impongano l'utilizzo di questa procedura in tutti pazienti HCV-positivi $(6,7)$. In questo studio è stato esaminato il quadro morfologico epatico presente in pazienti viremici (HCV-RNA+), che si presentavano al momento della valutazione per l'inserimento in lista trapianto con una normale funzione epatica, con o senza citolisi.

L'esame istologico ha mostrato la presenza di epatite cronica attiva (ECA) in un numero elevato di pazienti $(65 \%)$, ma nella quasi totalità, il grado di aggressività dell'epatite è risultato minimo/lieve. Solo in due casi $(3 \%)$ è stata documentata un'ECA di grado 
moderato e non sono mai stati osservati quadri di fibrosi a ponte o di cirrosi. Pertanto, l'esame istologico ha documentato, nel $97 \%$ dei casi, quadri di epatopatia lieve, tali da consentirne l'inserimento in lista trapianto.

Tuttavia, alcuni autori consigliano di attuare una terapia antivirale prima del trapianto in tutti i pazienti con ECA, indipendentemente dal grado e dallo stadio della malattia $(1,3)$. Questo approccio sarebbe giustificato dal fatto che il trattamento con interferone permetterebbe di superare questo fattore di rischio in molti pazienti, vista l'alta percentuale di risposta viremica persistente documentata nella popolazione dializzata (8). Comunque, non tutti concordano con questa strategia. Infatti, se da un lato l'infezione da HCV rappresenta la più importante causa di epatopatia dopo trapianto di rene (1) i suoi effetti negativi sulla sopravvivenza del paziente e del trapianto, si palesano solo nel lungo termine (9) e non sono peraltro confermati da tutti gli autori (10). Recenti lavori dimostrano come la progressione della fibrosi epatica secondaria ad epatite cronica da virus $C$ possa essere più lenta nel trapiantato di rene, rispetto al paziente immunocompetente (11) e possa addirittura regredire in una quota di pazienti trattati con ciclosporina (12, 13). Infine, vi è comune accordo sul fatto che, se si escludono le forme avanzate di epatite, la prognosi del paziente uremico $\mathrm{HCV}-\mathrm{Ab}$ positivo migliora dopo il trapianto, sia in termini di morbilità che di mortalità (14). Sulla base di questa evidenza, i più considerano come spartiacque per decidere l'idoneità o meno al trapianto, l'epatite aggressiva con grado di attività almeno moderato e/o con presenza di fibrosi portoportale (15). Non c'è dubbio quindi che l'ECA lieve non deve essere considerata una controindicazione all'inserimento in lista.

Nella nostra popolazione la probabilità di riscontrare all'esame istologico un quadro di ECA non è risultata correlata con le caratteristiche cliniche e bioumorali dei pazienti. Tuttavia, confrontando l'esito dell'esame istologico del gruppo di pazienti senza citolisi, rispetto a quello con citolisi, si osser- va che proprio in quest'ultimo sono strati osservati gli unici casi di ECA con attività moderata, quindi con caratteristiche tali da mettere in discussione l'idoneità al trapianto.

Sottolineiamo che la definizione di epatocitolisi che abbiamo utilizzato in questo studio è molto restrittiva. Infatti, è ampiamente documentato che nel paziente con insufficienza renale i livelli di transaminasi sono ridotti, probabilmente dimezzati rispetto ai range della popolazione generale (16). Basandoci su questa definizione abbiamo documentato la presenza di segni clinici di attività dell'epatite in circa il $26 \%$ dei pazienti HCV-Ab positivi. La prevalenza di ipertransaminasemia aumentava al 29\% quando cercata solo nei pazienti viremici. Questi valori sono sovrapponibili a quanto sinora documentato in letteratura (26-30\%) (17).

In conclusione, il quadro istologico epatico più frequentemente riscontrato tra i pazienti con HCV-RNA positività e normofunzione epatica, che accedono a una valutazione di idoneità al trapianto di rene, è l'ECA, ma con lesioni di grado lieve. Solo nei pazienti con ipertransaminasemia si possono osservare forme con aggressività maggiore (3\% dei casi). Ne consegue che, in assenza di alterazioni delle transaminasi, la biopsia epatica non rappresenta uno strumento indispensabile, ma lo diventa solo in presenza di epatocitolisi transitoria o persistente, nei casi in cui l'obiettivo sia stabilire l'idoneità al trapianto.

Qualora si decida invece di trattare tutti i pazienti con ECA, indipendentemente dalla gravità delle lesioni, l'esecuzione della biopsia epatica diventa allora indispensabile per una corretta selezione del paziente.

\section{BIBLIOGRAFIA}

1. Campistol JM, Esforzado N, Martinez J, et al. Efficacy and tolerance of interferon-(2b) in the treatment of chronic hepatitis $\mathrm{C}$ virus infection in haemodialysis patients: Pre- and post-renal transplantation assessment. Nephrol Dial Transplant 1999; 14: 2704-09.

2. Rao KV, Anderson WR, Kasiske BL, Dahl DC. Value of liver biopsy in the evaluation and management of chronic liver disease in renal transplant recipients. Am J Med 1993; 94: 214-50.

3. Morales JM, et al. Transplantation in the patient with hepatitis C. J Am Soc Nephrol 2000; 11 (7): 134353.

4. Pereira B, Levey A. Hepatitis C virus infection in dialysis and renal transplantation. Kidney Int 1997; 51: 981-99.

5. Fabrizi F, Bunnapradist $S$, et al. Treatment of HCV-related liver diseases after renal transplantation: modern views. Int J Artif Organs 2003; 26: 373-82.

6. Martin P, Carter D, et al. Histopathological features of hepatitis $\mathrm{C}$ in renal transplant candidates. Transplantation 2000; 69: 1479-84.

7. Ozdogan M, Ozgur O, et al. Histopathological impact of hepatitis virus infection in hemodialysis patients: should liver biopsy be performed before renal transplantation? Artif Organs 1997; 21: 355 .

8. Kamar N, Toupance O, et al. Evidence that clearance of hepatitis $\mathrm{C}$ virus RNA after interferon therapy in dialysis patients is sustained after renal transplantation. J Am Soc Nephrol 2003; 14: 2092-8.

9. Fabrizi F, Martin P, et al. Hepatitis $\mathrm{C}$ virus antibody status and survival after renal transplantation: metaanalysis of observational studies. Am J Transplant 2005; 5(6): 1452-61. 
10. Forman J, Tolkoff-Rubin N, et al. Hepatitis C, acute humoral rejection and renal allograft survival. J Am Soc Nephrol 2004; 15: 3249-55.

11. Alric L, Di-Martino V, et al. Long-term impact of renal transplantation on liver fibrosis during hepatitis $\mathrm{C}$ virus infection. Gastroenterology $2002 ; 123: 1494-9$.

12. Kamar N, Rostaing L, et al. Natural history of hepatitis $\mathrm{C}$ virus-related liver fibrosis after renal transplantation. Am J Transplant 2005; 5: 1704-12.

13. Nakagawa M, Sakamoto N, et al. Specific inhibition of hepatitis $\mathrm{C}$ virus replication by cyclosporin $\mathrm{A}$. Biochem Biophys Res Commun 2004; 313: 42-7.

14. Bloom RD, et al. Outcome of hepatitis $\mathrm{C}$ virus-infected kidney transplant candidates who remain on the waiting list. Am J Transplant 2005; 5: 139-44.

15. Gane E, Pilmore H. Management of chronic viral hepatitis before and after renal transplantation. Transplantation 2002; 27 (4): 427-37.

16. Fabrizi F, Lunghi G, Finazzi S, et al. Decreased serum aminotransferase activity in patients with chronic renal failure: impact on the detection of viral hepatitis. Am J Kidney Dis 2001; 38 (5): 1009-15.

17. Pol S, Romeo R, Zins B. Hepatitis $\mathrm{C}$ virus RNA in anti-HCV positive hemodialysed patients: significance and therapeutic implications. Kidney Int 1995; 47: 1412-8. 